\title{
Antitumor and antimetastatic activities of chloroform extract of medicinal mushroom Cordyceps taii in mouse models
}

\author{
Ru-Ming Liu' ${ }^{1}$, Xiao-Jie Zhang ${ }^{1}$, Gui-You Liang ${ }^{1}$, Yong-Fu Yang ${ }^{2}$, Jian-Jiang Zhong ${ }^{3^{*}}$ and Jian-Hui Xiao ${ }^{1 *}$
}

\begin{abstract}
Background: Cordyceps taii, an entomogenous fungus native to south China, is a folk medicine with varieties of pharmacological activities including anticancer effect. To validate the ethnopharmacological claim against cancer, the antitumor and antimetastatic activities of chloroform extract of C. taii (CFCT) were investigated in vivo.

Methods: The in vitro cytotoxic activities of CFCT against human lung cancer (A549) and gastric cancer (SGC-7901) cells were evaluated using the Sulforhodamine B (SRB) assay. In vivo anti tumor and antimetastatic activities, Kunming mice bearing sarcoma 180 and C57BL/6 mice bearing melanoma B16F10 were employed, respectively. The antitumor effects of CFCT were completely evaluated on the basis of the tumor weight, survival time, histologic analysis, and immune organ indices. The histopathological change, metastatic foci and malignant melanoma specific marker HMB45 in the lung tissue were detected for the evaluation of the antimetastatic activity of CFCT.

Results: CFCT exhibited dose- and time-dependent cytotoxicities against A549 and SGC-7901 cells with the IC 50 values of 30.2 and $65.7 \mu \mathrm{g} / \mathrm{mL}$, respectively. Furthermore, CFCT at a dose of 50 or $100 \mathrm{mg} / \mathrm{kg}$ could significantly inhibit the tumor growth in vivo and prolonged the survival time in two different models as compared with the model group, especially when combined with the CTX at a low dose rate. And it also increased spleen index of Kunming mice and thymus index of C57BL/6 mice. Meanwhile, histologic analysis illustrated that CFCT alone or in combination with CTX could induce tumor tissue necrosis of both models. In addition, CFCT at a dose of 50 or $100 \mathrm{mg} / \mathrm{kg}$ inhibited the lung metastasis of melanoma B16F10 in tumor-bearing C57BL/6 mice. The antimetastatic effect was also observed when CFCT was used in combination with CTX. In comparison to any other groups, CFCT at a dose of $100 \mathrm{mg} / \mathrm{kg}$ could effectively enhance the GSH-Px activities of various tissues in tumor-bearing C57BL/6 mice.
\end{abstract}

Conclusions: These findings demonstrate that CFCT has potent in vivo antitumor and antimetastatic activities, and may be helpful to the development of anticancer chemopreventive agents from C. taii.

Keywords: Chinese traditional medicine, Cordyceps taii, Anticancer active ingredients, Antimetastatic activity, Tumorburdened mouse model

\footnotetext{
* Correspondence: jjzhong@sjtu.edu.cn; jhxiao@yahoo.com

${ }^{3}$ State Key Laboratory of Microbial Metabolism, Joint International Research Laboratory of Metabolic \& Developmental Sciences, and School of Life Sciences \& Biotechnology, Shanghai Jiao Tong University, Shanghai 200240, China ${ }^{1}$ Guizhou Center for Translational Medicine \& Laboratory of Cell Engineering, Affiliated Hospital of Zunyi Medical University, Zunyi 563000, China Full list of author information is available at the end of the article
} 


\section{Background}

Due to substantial morbidity and high mortality, cancer is considered as the second "killer" in the world [1]. A latest global cancer trend report by the World Health Organization shows that new cancer cases worldwide will remain a rapid increase, and reach more than 19 million a year by 2025 [2]. Cancer is a major global health crisis accordingly, and urgently needs effective prevention measures to curb the disease. Although the development of biological therapies have been used as new strategies for cancer treatment in recent years, surgery, radiotherapy and chemotherapy are still the three most frequently used therapies in the world. In comparison with surgery and radiotherapy, chemotherapy possess some advantages like systemic treatment and not causing significant physical damage, and it is recommended for patients with extrapelvic metastases or recurrent disease who are not candidates for the other two treatments [3]. However, long-term use of single-agent or combination chemotherapy usually results in severe side effects and resistance [4]. Obviously there is an urgent demand for exploring new chemotherapy drugs with high efficiency and low toxicity. The discovery of taxol and camptothecin lights up the hope for searching new anticancer drugs from natural sources.

The genus Cordyceps, an entomogenous fungus with a wide variety of pharmacological properties, is a well-known and valuable source of traditional Chinese medicine. The applications of Cordyceps in China and other Eastern Asian countries suggest that it may be used for cancer prevention and treatment [5]. Furthermore, current Cordyceps has received considerable attention worldwide as a potential source of anticancer drugs [6, 7]. Cordyceps taii is a folk medicine native to south China [8]. Previously, polysaccharides from $C$. taii were found to display immunomodulatory, antitumor, and antimutagenic activities [8]. Our recent findings suggest that $C$. taii polysaccharides are a promising source of natural antioxidant and antiaging agents [9]. Further pharmacological experiments indicate that C. taii has broad-spectrum antimicrobial effects including potent antibacterial and antifungal activities, and the active ingredients were found to be enriched in non-polar fractions extracted with organic solvents such as chloroform, ethyl acetate, and acetone $[10,11]$. However, the anticancer potential of these non-polar fractions of $C$. taii has never been investigated. In view of the broad spectrum of therapeutic potentials of Cordyceps spp., the aim of the present study was to assess the antitumor and antimetastatic activities of chloroform extract of C. taii (CFCT) in vivo, and to promote the further development of anticancer active ingredients from C. taii.

\section{Methods}

\section{Chemicals and reagents}

Dimethylsulfoxide (DMSO), sulforhodamine B (SRB), Tris, trypsin, and trypan blue were purchased from
Sigma-Aldrich (USA). The RPMI-1640 medium, 4-(2hydroxyethyl)-1-piperazineethanesulfonic acid (HEPES), $L$-glutamine, and fetal calf serum were purchased from Gibco (USA). Cyclophosphamide (CTX) was purchased from Hengrui Pharmaceuticals, Co. Ltd. (Nanjing, China). Olive oil was purchased from Zhongqi Huaye Inc. (Beijing, China). Histo-clear agent was purchased from ESM Inc. (USA). HMB45 anti-melanoma antibody was purchased from Abcam, PV-6002 non-biotin two-step IHC detection kit (Horseradish peroxidase-conjugated goat antimouse IgG secondary antibody) was supplied by Zhongshan Golden Bridge Biotechnol. Co. Ltd. (Beijing, China), DAB Horseradish Peroxidase Color Development Kit was purchased from Beyotime Ins. Biotechnol (Haimen, China). Glutathione peroxidase (GSH-Px) antioxidative enzyme detection kit was from Jiancheng Bioengin Ins (Nanjing, China). All chemicals used were of analytic grade.

\section{Medicinal fungus, cultivation, and mycelia preparation}

The voucher specimens of C. taii (strain GYYA 0601) were deposited at the Laboratory of Microbial Resources \& Drug Development, Center for Translational Medicine, Affiliated Hospital of Zunyi Medical University, Guizhou Province, China. The mycelia of C. taii were cultured and harvested as previously described [12, 13]. Subsequently, the mycelia were lyophilized and grinded (60 mesh to 100 mesh) for later experiments.

\section{Preparation of active fraction}

The dried mycelia power of C. taii (4400 g) was extracted five times with $25 \mathrm{~L}$ of $80 \%(\mathrm{v} / \mathrm{v})$ aqueousmethanol solution by hot soaking. The collected extract solutions were filtered through a $0.45 \mu \mathrm{m}$ pore-size filter, and their solvents were then removed at $40{ }^{\circ} \mathrm{C}$ by a rotary evaporator under vacumm to yield a brown $\mathrm{MeOH}$ extract (2252 g). Subsequently, the extract was resuspended in hot water. The suspension was extracted five times with equal volumes of petroleum ether (b.p.60-90 ${ }^{\circ}$ ) to yield the petroleum ether extract, and the aqueous residue was further extracted five times with equal volumes of chloroform. Finally, the combined chloroform layers were evaporated using a rotary evaporator under reduced pressure to yield the chloroform extract of C. taii (CFCT, 210 g). Stock solution of CFCT was prepared in DMSO and stored at $-20{ }^{\circ} \mathrm{C}$. Further dilution was made with medium just before use, and the final concentration of DMSO was less than $0.1 \%(\mathrm{v} / \mathrm{v})$.

\section{Cell line and culture}

The human lung cancer cell line A549, gastric cancer cell line SGC-7901, and the mice cancer cell lines such as sarcoma 180 and melanoma B16F10 were purchased from the Cell Bank of Type Culture Collection of the Chinese Academy of Sciences (Shanghai, China). All the 
above four cell lines were grown in RPMI 1640 medium supplemented with $10 \%$ fetal bovine serum, $25 \mathrm{mM}$ HEPES buffer, and $2 \mathrm{mML}$-glutamine in a humidified incubator (Thermo, USA) preset to $37{ }^{\circ} \mathrm{C}$ and $5 \% \mathrm{CO}_{2}$. Cells at the logarithmic growth stage were used for all experiments.

\section{Animals}

Kunming (KM) mice and C57BL/6 mice (male; age: 5 to 7 weeks; weight: $18.0 \pm 2.0 \mathrm{~g}$ ) were provided by the Experimental Animal Center of the Third Military Medical University in Chongqing, China (Animal License No. SCXK (YU) 2007-2006). The animals were kept in a standard laboratory environment and fed with sterile pellets and water ad libitum. The laboratory animal protocol for this study was approved by the Zunyi Medical College Committee for the Control and Supervision of Experimental Animals. All experimental animals were bred for $7 \mathrm{~d}$ before use.

\section{Assay of cytotoxic activity}

The SRB colorimetric method was employed, as previously described, to measure the cytotoxic activities of CFCT against human cancer cells, including the dose- and timeresponses [14]. CFCT was dissolved in DMSO and stored at $-20^{\circ} \mathrm{C}$. The thawed samples were immediately diluted in RPMI 1640 medium to reach a concentration of $1 \mathrm{mg} / \mathrm{mL}$ before further use. For the analysis of the dose-response, cancer cells were seeded into 96-well plates at a density of $6 \times 10^{3}$ cells in $100 \mu \mathrm{L}$ complete medium per well and were incubated at $37{ }^{\circ} \mathrm{C}$ for $24 \mathrm{~h}$. Eight concentrations of CFCT $(100 \mu \mathrm{L})$ were added to each well, and the final concentrations were $1.9,3.9,7.8,15.6,31.25,62.5,125.0$, and $250.0 \mu \mathrm{g} / \mathrm{mL}$, respectively. These samples were further incubated for $48 \mathrm{~h}$. A blank control group (culture media only), a negative control group (cells cultured in the media), a positive control group (cells were treated with $25 \mu \mathrm{g} / \mathrm{mL}$ DDP), and a solvent control group (cells treated with DMSO at the same concentration as the treatment drug group) were all placed in the same 96-well plate. After $48 \mathrm{~h}$ of drug exposure, its cytotoxic effect was detected using a SRB colorimetric method as previously reported [14]. The absorbance data were exported into a Microsoft Excel spreadsheet (Microsoft) for further analysis. Cell densities were obtained by correcting the absorbance based on the blank controls. Cell survival and/or cell growth inhibitory rate was calculated as the percentage absorbance compared with that of the negative control. Likewise, cancer cells were exposed to $40 \mu \mathrm{g} / \mathrm{mL}$ of CFCT for $8,20,32,44,56,68$ and $76 \mathrm{~h}$, respectively, for analysis of the time response.

\section{Antitumor activity of CFCT in subcutaneous sarcoma 180-bearing mice}

The sarcoma-180 (S180) solid tumor model was empolyed to assess the antitumor effect of the CFCT in vivo on the basis of the tumor growth and host survival. Tumor cells were harvested from the peritoneal cavity of KM mice with a 10-day-old S180 ascitic tumor under the sterile condition, washed twice with sterile saline, and suspended in the sterile saline at a density of $1 \times 10^{7}$ cells/mL. KM mice were then inoculated subcutaneously (s.c.) $0.2 \mathrm{~mL}$ of tumor cells suspension per mouse into their right hind limbs on day 0 . Subsequently, the mice were randomly divided into seven groups $(n=16)$. CFCT was dissolved in a certain amount of olive oil. The CFCT treatment groups were administered i.p. daily to the mice at doses of 20, 50 and $100 \mathrm{mg} / \mathrm{kg}$ (LG, MG and HG groups) for 7 consecutive days, starting $24 \mathrm{~h}$ after tumor transplantation. A combined administration group (CG group, $20 \mathrm{mg} / \mathrm{kg}$ of CFCT $+20 \mathrm{mg} / \mathrm{kg}$ of CTX, once daily), a positive control group (PG group, $20 \mathrm{mg} / \mathrm{kg}$ of CTX, once daily), and a negative control group (SG group, mice were treated with olive oil at the same volume as the CFCT treatment group) were also employed on the same schedule. The Model group was treated with saline only. The tumor size was measured with digital calipers every day, and its volume $\left(\mathrm{cm}^{3}\right)$ was calculated as the (length $x$ width $\left.^{2}\right) / 2$. Two independent experiments were performed for each treatment with eight mice per group. On day 8 , eight mice from each group were anaesthetized and sacrificed by cervical dislocation, and their bodies were weighed. Simultaneously, their solid tumor, thymus and spleen were quickly removed and weighted, respectively. The tumors were fixed in $10 \%$ paraformaldehyde for at least $24 \mathrm{~h}$, and then were embedded in paraffin under vaccum. While the rest were allowed to live to a natural death, and the death time was recorded to calculate the median survival time (MST). The percentage increase in life span (ILS) of tumor hosts was calculated on the basis of mortality of the experimental mice: $\mathrm{MST}=\Sigma$ Survival time of each mouse in a group/Total number of mice; $\%$ ILS $=($ MST of treated group/MST of control group $) \times$ 100. The immune organ indices were defined as the thymus and/or spleen weight relative to body weight. Spleen (thymus) index $=$ Spleen (thymus) weight/body weight $x$ $100 \%$. The tumor inhibitory ratio $=$ (the average tumor weight of model group - the average tumor weight of treatment group)/the average tumor weight of model group $\times 100 \%$.

\section{Antitumor and antimetastatic activities of CFCT in subcutaneous melanoma B16F10-bearing mice}

Cultured murine melanoma B16F10 cells during the exponential phase of growth were harvested by trypsinization, washed, and suspended at $1.5 \times 10^{6} \mathrm{cells} / \mathrm{mL}$ in RPMI-1640 medium supplemented with $10 \%$ FBS. C57BL/6 mice $(\mathrm{n}=112)$ were injected s.c. with $2.25 \times$ $10^{5}$ B16F10 cells per mouse into the lower right groin on day 0 , and were then randomly assigned into seven 
groups $(\mathrm{n}=16)$. After the implantation of tumor, $0.2 \mathrm{~mL}$ CFCT (20, 50 and $100 \mathrm{mg} / \mathrm{kg}$ ) was administered i.p. once every other day for two weeks in the treatment group. As described above, two independent experiments were performed for each treatment with eight mice per group, and the CG, PG, SG, and Model groups were also given on the same schedule. On day 15, eight mice from each group were anaesthetized and sacrificed by picking off the eyeballs, and their blood sample, liver, brain were quickly harvested. All tumor, thymus, spleen and lung were also removed and weighted for the assession of antitumor and antimetastic activities and sideeffects. Invasive metastases to the lung were observed manually. The rest of mice were used as assessing the survival time.

\section{Histological examination and immunohistochemistry staining}

For histopathology studies, all tumors were washed by normal saline, fixed by $10 \%$ paraformaldehyde in phosphate buffer saline, successively dehydrated in solutions containing an increasing percentage of ethyl alcohol (70, 80, 95 and $100 \%)$, embedded in paraffin under a vacuum, cut into $5 \mu \mathrm{m}$-thick sections, deparaffinized in histo-clear agent, and stained with Harris hematoxylineosin (HE staining).

For immunohistochemical staining, lung tissues in C57BL/6 mouse were fixed for at least $24 \mathrm{~h}$ by Bouin soultion. Lung tissue sections of $5 \mu \mathrm{m}$-thick were dried overnight at $65{ }^{\circ} \mathrm{C}$ and deparaffinized in histo-clear. The sections were rehydrated through graded alcohols into water. After rehydrating, antigen retrieval was carried out by heating for $20 \mathrm{~min}$ at $100{ }^{\circ} \mathrm{C}$ in $10 \mathrm{mM}$ citrate buffer (pH 6.0). Endogenous peroxidase activity was blocked with $3 \% \mathrm{H}_{2} \mathrm{O}_{2}$ in methanol for $10 \mathrm{~min}$ at room temperature and non-specific binding of reagents was quenched by $10 \%$ normal goat or rabbit serum. After rinsing with distilled water for $5 \mathrm{~min}$, the sections were incubated at $4{ }^{\circ} \mathrm{C}$ to stay overnight with primary anti-mouse HMB45 monoclonal antibody. The sections were rinsed with PBS again for $2 \mathrm{~min}$, and then were incubated at $37^{\circ} \mathrm{C}$ for $30 \mathrm{~min}$ with horseradish peroxidase-conjugated goat anti-mouse IgG secondary antibody. After washing by PBS, the sections were stained using a DAB kit and observed under microscope. Appropriate positive and negative controls without primary antibody were included.

\section{Analysis of the antioxidant endogenous enzyme GSH-Px}

The defense effect of CFCT on antioxidant-related endogenous enzyme GSH-Px was investigated. Blood samples were collected from the orbital venous plexuses of the mice under anesthesia. The brains and livers were rapidly excised and thoroughly washed to clear off blood. These organs were immediately transferred to ice-cold saline and homogenized (10\%) in cold saline (about $4{ }^{\circ} \mathrm{C}$ ). The blood and homogenate tissues were centrifuged at $3000 \times \mathrm{g}$ and $4{ }^{\circ} \mathrm{C}$ for $20 \mathrm{~min}$. GSH-Px in the supernatants was assessed using the respective detection kits as previously described [9].

\section{Statistical analysis}

For each measured drug concentration, there were five to eight identical wells in the 96-well culture plates. All experiments were performed at least three times. The experimental data were statistically analyzed using the SPSS (version 13.0) software, and the data were expressed as the means with their corresponding standard errors. When appropriate, statistical significance was analyzed using a two-tailed Student's $t$-test. Differences were considered statistically significant if $P<0.05$.

\section{Results}

\section{Cytotoxic activity of CFCT against human cancer cells in} vitro

The cytotoxic activities of CFCT against two different cancer cell lines, i.e. human lung carcinoma A549 cells and human gastric carcinoma SGC-7901 cells, were displayed in Fig. 1. CFCT exerted potent cytotoxic activities in a dose-dependent manner at the dose range from 1.9 to $250.0 \mu \mathrm{g} / \mathrm{mL}$ after $48 \mathrm{~h}$ of exposure (Fig. 1a). The $\mathrm{IC}_{50}$ values of CFCT against cancer cells were calculated to be $30.2 \pm 2.6$ and $65.7 \pm 5.3 \mu \mathrm{g} / \mathrm{mL}$ for A549 and SGC-7901 cells, respectively. Therefore, the A549 cells showed about two-fold sensitivity toward CFCT in comparision to SGC7901 cells. As shown in Fig. 1b, the cytotoxic activities of CFCT against A549 cancer cells presented approximately in a time-dependent manner within the time range tested at a dosage of $40 \mu \mathrm{g} / \mathrm{mL}$, but a non-time-dependent manner for SGC-7901 cells.

\section{Antitumor effect of CFCT in S180 tumor-bearing KM mice}

As shown in Table 1, the tumor weight of HG, MG, and CG groups was decreased significantly compared with that of the model group. However, no significant difference of tumor weight was observed between LG and model groups. The inhibitory ratio of HG, MG and CG groups was $57.9 \pm 8.9 \%, 33.6 \pm 12.8 \%$, and $69.4 \pm 9.7 \%$ (Fig. 2a), respectively, which presented significant differences in comparison with model control $(P<0.01)$. Furthermore, the inhibitory ratio of the positive control $(56.1 \pm 9.1 \%)$ was also lower than both HG and CG groups. Further histopathological analysis of tumor tissue by HE staining showed a large number of necrotic cancer cells or tissues (blue arrow denote necrotic cells) in the HG, MG and CG groups (Fig. 2b). Accordingly, these data indicated that CFCT could significantly inhibit tumor growth in S180 tumor-bearing KM mice. 

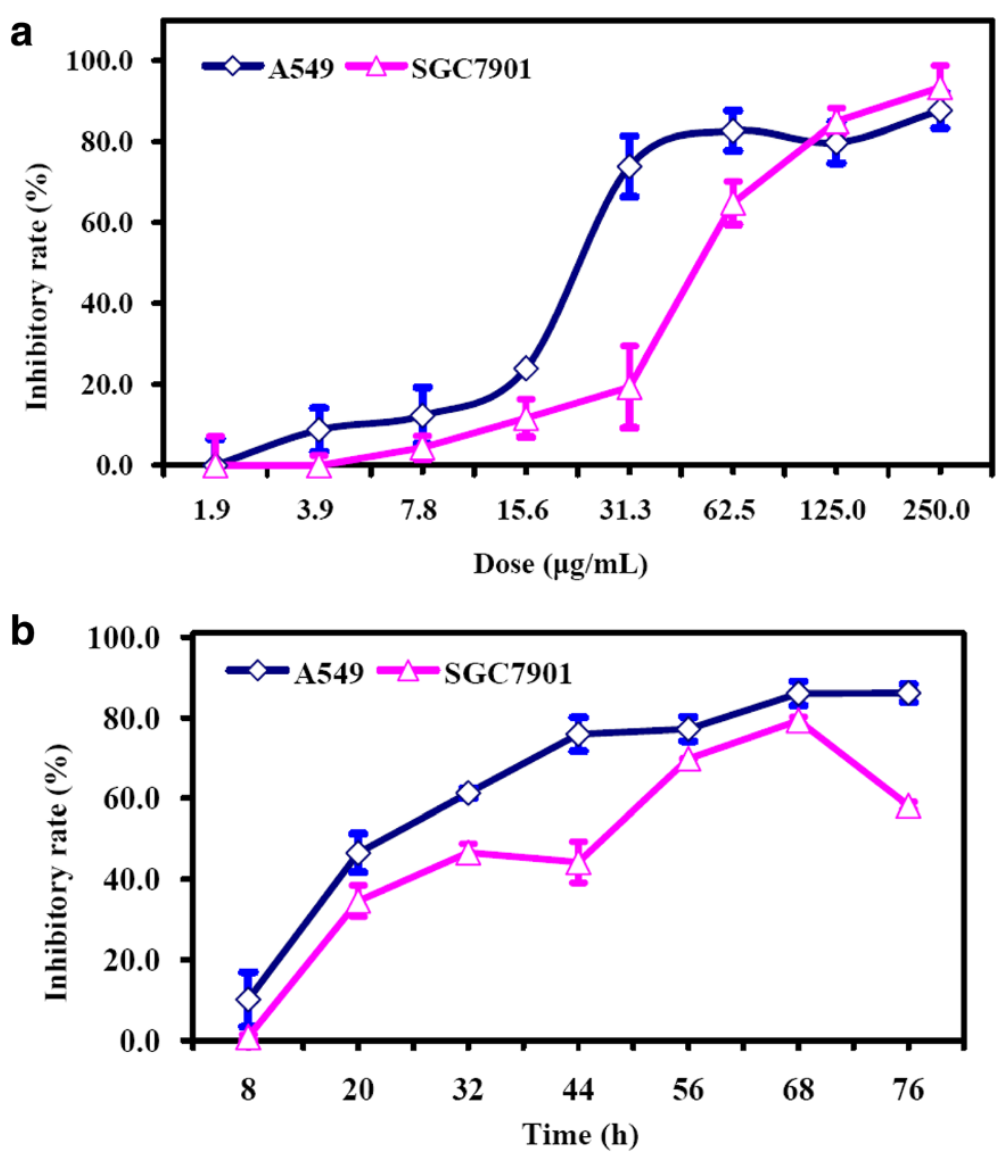

Fig. 1 Cytotoxicity of CFCT against human lung carcinoma A549 cells and human gastric carcinoma SGC-7901 cells. a A549 cells and SGC-7901 cells treated with different concentrations of CFCT for 48 h; b A549 cells and SGC-7901 cells treated with CFCT (40 $\mu \mathrm{g} / \mathrm{mL})$ for a different period (8-76 h). Data were shown as mean \pm SD $(n=3)$

\section{Antitumor effect of CFCT in melanoma B16F10-bearing C57BL/6 mice}

CFCT could inhibit the tumor growth of murine metastatic melanoma B16F10-bearing mice in comparison with the model group, especially in the HG and CG groups (Table 2). As shown in Fig. 3a, it resulted in more than $30 \%$ inhibition in both HG group $(P<0.05)$ and CG group $(P<0.01)$ compared with the model group. However, no significant difference of tumor weight was observed between LG group and the model group. Further histopathological analysis of tumor tissue with HE staining displayed a large number of necrotic cancer cells or tissues (blue arrow denote necrotic cells) in the HG and CG groups (Fig. 3b). Therefore, these data suggested that CFCT could moderately inhibit tumor growth in B16F10-bearing C57BL/6 mice.

\section{Immune organ index and survival prolongation of} tumor-bearing mice after CFCT treatment

Thymus and spleen are the primary immune organs and directly affect the organism's immune function [15]. The

Table 1 Effects of CFCT on tumor weight and immune organ indices on day 8 in 5180 tumor-bearing KM mice

\begin{tabular}{|c|c|c|c|c|}
\hline Group & Dosage $(\mathrm{mg} / \mathrm{kg})$ & Tumor weight (g) & Spleen index (mg/g) & Thymus index $(\mathrm{mg} / \mathrm{g})$ \\
\hline Model & - & $0.97 \pm 0.19$ & $4.683 \pm 1.149$ & $2.994 \pm 0.702$ \\
\hline$P G$ & 20.0 & $0.42 \pm 0.09^{* *}$ & $5.600 \pm 0.667$ & $3.060 \pm 0.397$ \\
\hline SG & - & $0.91 \pm 0.23$ & $4.484 \pm 1.064$ & $2.611 \pm 0.375$ \\
\hline $\mathrm{HG}$ & 100.0 & $0.41 \pm 0.09^{* *}$ & $6.288 \pm 0.983^{*}$ & $2.512 \pm 0.190^{*}$ \\
\hline MG & 50.0 & $0.64 \pm 0.12^{* *}$ & $5.750 \pm 0.907^{*}$ & $2.230 \pm 0.293^{* *}$ \\
\hline LG & 20.0 & $0.83 \pm 0.26$ & $4.864 \pm 0.584$ & $2.587 \pm 0.230^{*}$ \\
\hline CG & $(\mathrm{LG}+\mathrm{PG})$ & $0.30 \pm 0.09^{* *}$ & $5.751 \pm 1.146^{*}$ & $2.723 \pm 0.410$ \\
\hline
\end{tabular}

Values are mean $\pm \mathrm{SD}(\mathrm{n}=8) .{ }^{* *} P<0.01,{ }^{*} P<0.05$ vs. model control group. All groups as described with Fig. 2 

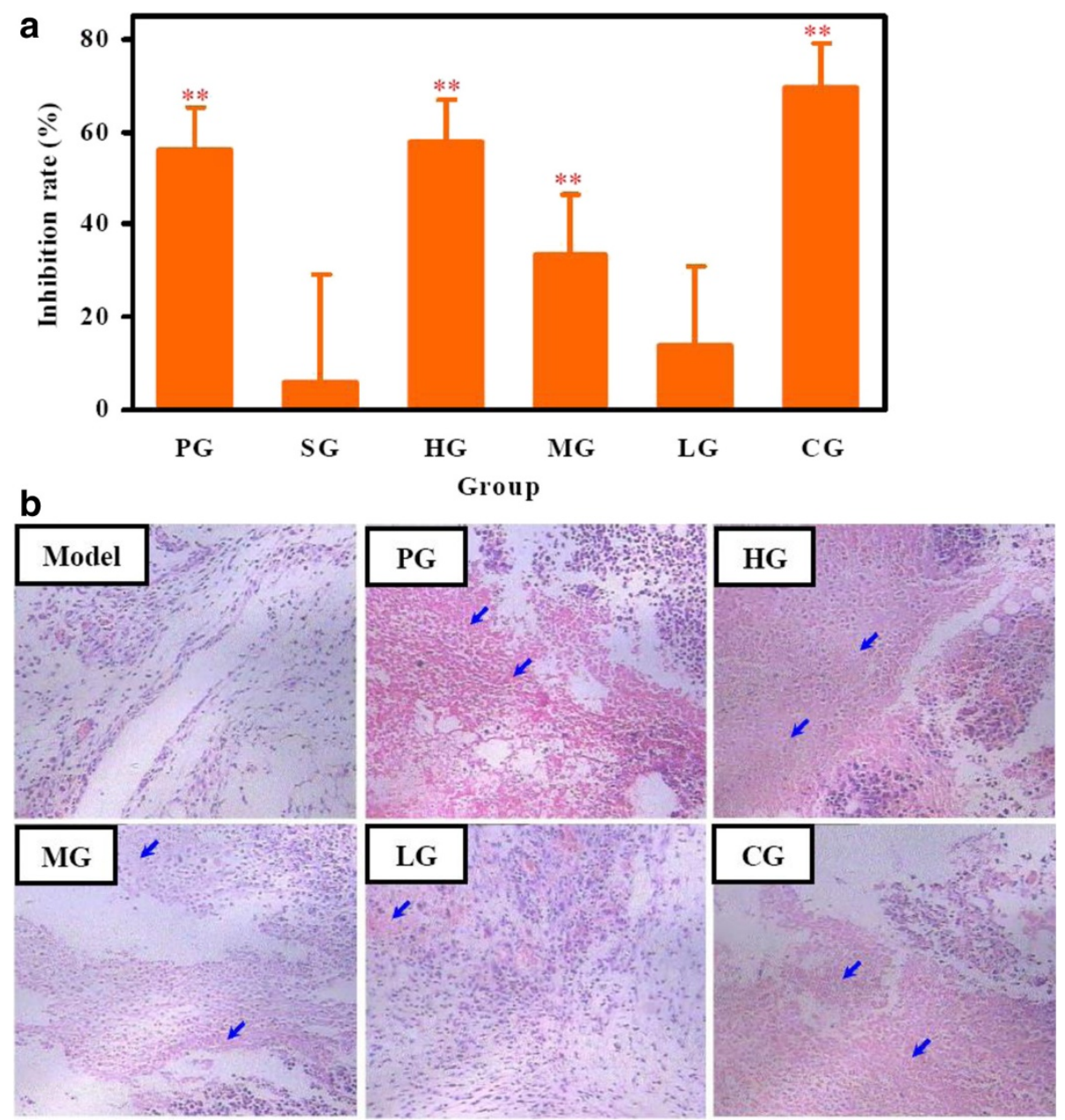

Fig. 2 Antitumor effect of CFCT in S180 tumor-bearing KM mice. a Inhibitory rate in treatment groups. Values were expressed as mean \pm SD $(n=8)$. The inhibitory ratio = (the average tumor weight of model group - the average tumor weight of treatment group)/the average tumor weight of model group $\times 100 \%$; b Representative HE staining sections from different groups. Blue arrows denote necrotic tissues. ${ }^{* *} P<0.01$ vs. model group. Model group, mice were treated with olive oil at the same volume as the CFCT treatment group; PG, mice were treated with cytoxan $(20 \mathrm{mg} / \mathrm{kg})$; SG, mice were treated with saline at the same volume as the CFCT treatment group; HG, mice were treated with CFCT $(100 \mathrm{mg} / \mathrm{kg}) ; \mathrm{MG}$, mice were treated with CFCT (50 mg/kg); LG, mice were treated with CFCT $(20 \mathrm{mg} / \mathrm{kg})$; CG, mice were treated with cytoxan $(20 \mathrm{mg} / \mathrm{kg})$ and CFCT $(20 \mathrm{mg} / \mathrm{kg})$

Table 2 Effects of CFCT on tumor weight and immune organ indices on day 15 in melanoma B16F10-bearing C57BL/6 mice

\begin{tabular}{lllll}
\hline Group & Dosage $(\mathrm{mg} / \mathrm{kg})$ & Tumor weight $(\mathrm{g})$ & Spleen index $(\mathrm{mg} / \mathrm{g})$ & Thymus index $(\mathrm{mg} / \mathrm{g})$ \\
\hline Model & - & $2.07 \pm 0.29$ & $9.648 \pm 1.992$ & $1.133 \pm 0.288$ \\
PG & 20.0 & $1.04 \pm 0.48^{* *}$ & $8.067 \pm 1.300$ & $1.955 \pm 0.460^{* *}$ \\
SG & - & $2.12 \pm 0.31$ & $9.152 \pm 1.783$ & $0.954 \pm 0.291$ \\
HG & 100.0 & $1.42 \pm 0.47^{*}$ & $6.858 \pm 1.321^{*}$ & $1.800 \pm 0.521^{*}$ \\
MG & 50.0 & $1.62 \pm 0.26^{*}$ & $7.180 \pm 1.437^{*}$ & $1.603 \pm 0.427^{*}$ \\
LG & 20.0 & $1.76 \pm 0.25$ & $7.080 \pm 2.369^{*}$ & $1.164 \pm 0.390$ \\
CG & $(\mathrm{LG}+\mathrm{PG})$ & $1.01 \pm 0.32^{* *}$ & $8.284 \pm 1.806$ & $1.136 \pm 0.466$ \\
\hline
\end{tabular}

Values are mean $\pm \mathrm{SD}(\mathrm{n}=8) .{ }^{*} P<0.01,{ }^{*} P<0.05$ vs. model control group. All groups as described with Fig. 2 

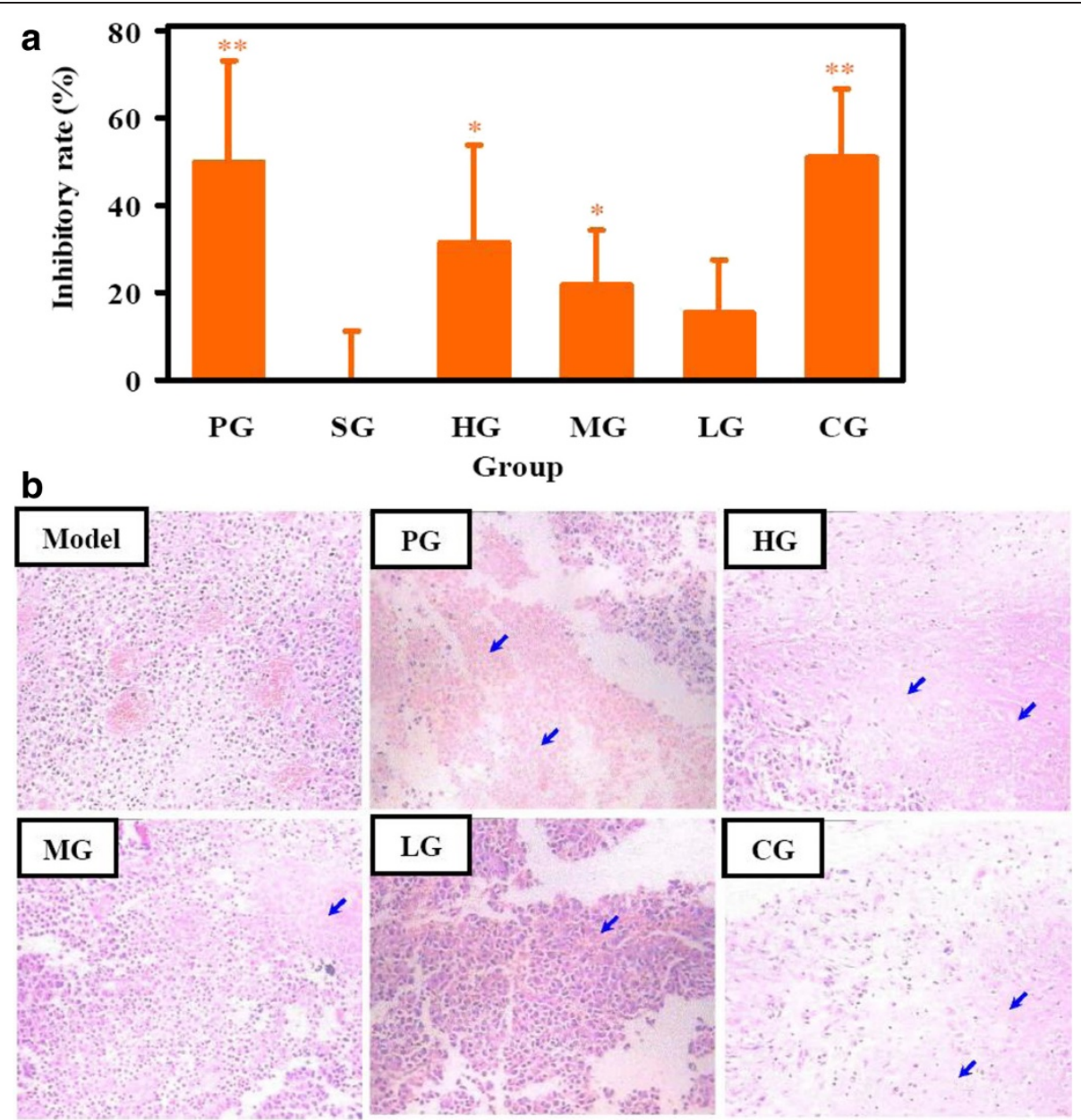

Fig. 3 Antitumor effect of CFCT in melanoma B16F10-bearing C57BL/6 mice. a Inhibitory rate in treatment groups. Values were expressed as mean $\pm S D(n=8)$. The inhibitory ratio = (the average tumor weight of model group - the average tumor weight of treatment group)/the average tumor weight of model group $\times 100 \%$; b Representative HE staining sections from different groups. Blue arrows denote necrotic tissues. ${ }^{*} P<0.05,{ }^{* *} P<0.01$ vs. model group. All groups as described with Fig. 2

effects of CFCT on the thymus index and spleen index of both tumor-bearing mouse models were shown in Table 1 and Table 2. Compared to the model control group, the spleen index of KM mice and the thymus index of C57BL/ 6 mice in the MG and HG groups were significantly increased $(P<0.05)$. However, CFCT at all dosages tested had obvious inhibitory effects on the thymus index of $\mathrm{KM}$ mice and the spleen index of C57BL/ 6 mice $(P<0.05)$.

The beneficial effects of CFCT on tumor-bearing mouse models were also reflected in the survival time. Survival times of the tumor-bearing mice in the MG, HG and CG groups were significantly prolonged for both tumorbearing models compared with the model control group (Table 3, Table 4, and Fig. 4, $P<0.01$ ). The extension rate of lifespan of HG group was slightly lower than that of positive control group PG, but the extension rate of lifespan of CG group was higher than that of PG group (Table 3, Table 4, and Fig. 4).

\section{Antimetastatic activity of CFCT in melanoma B16F10-bearing C57BL/6 mice}

Malignant melanoma, with high metastasis risk, often transfers to the lung tissue in C57BL/6 mice by blood metastasis, and is commonly used as a model of tumor metastasis [16]. Histopathological section of tumor-bearing C57BL/6 mice lung tissue with HE staining showed obviously metastatic foci of melanoma B16F10 (blue arrow) in the model and LG groups (Fig. 5), particularly in the model group. But metastatic foci were not observed in other groups.

HMB45, molecular weight of $7000 \mathrm{kDa}$, is a specific protein marker for melanoma cells, and mainly distributed in the cytoplasm [17]. Further, the expression of HMB45 in the lung tissue of tumor-bearing C57BL/6 mice was assessed by the immunohistochemical staining method. As shown in Fig. 6, the cytoplasm was basically dyed claybank (red arrow), which indicated the aggregation of 
Table 3 Effect of CFCT on survival rate in S180 tumor-bearing KM mice

\begin{tabular}{llll}
\hline Group & Dosage $(\mathrm{mg} / \mathrm{kg})$ & Survival time $(\mathrm{d})$ & Survival prolongation rate $(\%)$ \\
\hline Model & - & $33.2 \pm 1.7$ & - \\
PG & 20.0 & $42.5 \pm 1.4^{* *}$ & $26.39 \pm 0.82^{* *}$ \\
SG & - & $34.1 \pm 1.7$ & $1.49 \pm 0.11$ \\
HG & 100.0 & $42.1 \pm 1.6^{* *}$ & $25.28 \pm 0.14^{* *}$ \\
MG & 50.0 & $39.1 \pm 1.1^{* *}$ & $16.36 \pm 1.68^{* *}$ \\
LG & 20.0 & $36.4 \pm 1.2$ & $8.18 \pm 1.49$ \\
CG & $(L G+P G)$ & $42.9 \pm 2.0^{* *}$ & $27.51 \pm 0.80^{* *}$ \\
\hline
\end{tabular}

Values are mean $\pm S D(n=8) .{ }^{* *} P<0.01$ vs. model control group. All groups as described in Fig. 2

HMB45 positive cells in the solid tumor tissue (TG) of model group. Consistently, a clay-bank lump (red arrow), meaning small metastatic foci, was found in the lung tissue of model group, and it was different from the surrounding normal lung tissue. Only a few HMB45 positive cells were observed in the lung tissue of LG group, and they were not found in the MG, HG and CG groups. In addition, the HMB45 positive cells were more spread out in comparison to the model group and metastatic foci were not found in the lung tissue of solvent group (SG).

Based on the above results of HE and HMB45 immunohistochemistry staining, the HG and MG groups of CFCT could effectively inhibit the lung metastasis of malignant melanoma B16F10 in C57BL/6 mice.

\section{Effect of CFCT on the GSH-Px activity in melanoma B16F10-bearing C57BL/6 mice}

Studies have shown that enhancement of antioxidant capacity is one of the main action mechanism of anticancer drugs [18]. In the present study, the effects of CFCT on the activities of endogenous antioxidant enzyme GSH-Px in the blood, brain, and liver tissues were investigated in melanoma B16F10-bearing C57BL/6 mice (Fig. 7). Compared with the model group, CFCT at $100 \mathrm{mg} / \mathrm{kg}$ (HG group) evidently enhanced the enzyme activities of GSH-Px in all tested tissues $(P<0.05$ or $P<$ $0.01)$. In addition, the MG $(P<0.05)$ and CG $(P<0.01)$ groups of CFCT only increased the enzyme activities of GSH-Px in blood.

\section{Discussion}

Cordyceps is a potential source for the discovery of anticancer drugs. It has been well documented that Cordyceps-derived extracts and/or compounds showed potent inhibitory activities against different cancer cell lines in vitro through different mechanisms as reviewed previously [6]. However, in vitro methods are susceptible to falsepositive and false-negative results [19]. Therefore, it is emphasized that antitumor efficacy testing in rodents should be used to predict the possible clinical response. Until now, only a few of Cordyceps-derived polysaccharides and/or water extracts have been investigated for their antitumor potentials in the tumor-bearing animal models [20-22], and in vivo antitumor effects of Cordyceps-derived lipophlic extracts and/or chemical entities have yet to be elucidated.

This study showed that CFCT, a lipophlic extract of C. taii, possessed moderate cytotoxicity against human cancer cell lines in vitro, and furthermore it could remarkably inhibit tumor growth in S180 tumor-bearing KM mice and melanoma B16F10-bearing C57BL/6 mice. CFCT also prolonged the survival time and increased the survival rate in both models. The histopathological results indicated that each administration group of CFCT could effectively inhibit the tumor growth and nuclear fission, and lead to necrosis of tumor tissue, especially for the HG and CG groups. It is known that both the innate and the adaptive immune systems are active against cancers [23]. Thymus is a top central immune

Table 4 Effect of CFCT on survival rate in B16-bearing C57BL/6 mice

\begin{tabular}{llll}
\hline Group & Dosage $(\mathrm{mg} / \mathrm{kg})$ & Survival time $(\mathrm{d})$ & Survival prolongation rate $(\%)$ \\
\hline Model & - & $32.13 \pm 1.46$ & - \\
PG & 20.0 & $38.75 \pm 1.04^{* *}$ & $20.62 \pm 1.32^{* *}$ \\
SG & - & $32.38 \pm 1.41$ & $0.78 \pm 0.16$ \\
HG & 100.0 & $38.13 \pm 1.55^{* *}$ & $18.68 \pm 0.30^{* *}$ \\
MG & 50.0 & $35.50 \pm 1.69^{* *}$ & $10.51 \pm 0.72^{* *}$ \\
LG & 20.0 & $33.00 \pm 2.14$ & $2.72 \pm 2.12$ \\
CG & $(L G+P G)$ & $39.13 \pm 1.13^{* *}$ & $21.79 \pm 1.03^{* *}$ \\
\hline
\end{tabular}

Values are mean $\pm S D(n=8) .{ }^{* *} P<0.01$ vs. model control group. All groups as described in Fig. 2 

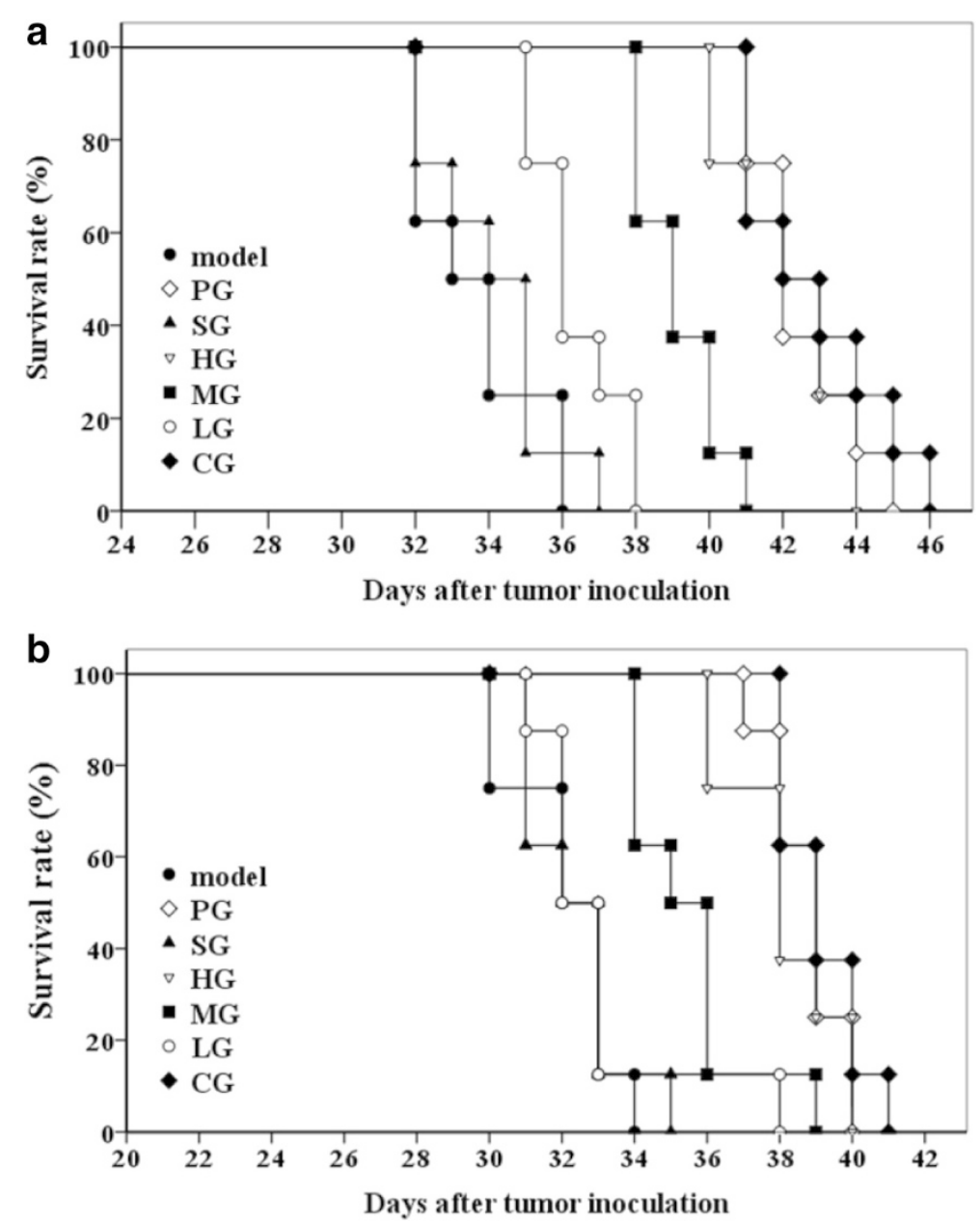

Fig. 4 Survival prolongation of tumor-bearing models after CFCT treatment. a Survival time of S180 tumor-bearing KM mice; b Survival time of melanoma B16F10-bearing C57BL/6 mice. Graph shows Kaplan-Meier survival curves for tumor-bearing mice treated with different groups. Survival was significantly longer in LG-treated mice vs. model group (log-rank test, $P<0.05)$. Data were presented as mean $\pm S D(n=8)$. All groups were the same as described in the legends of Fig. 2

organ, and spleen is one of the biggest peripheral immune organs $[24,25]$. The viscera indices of thymus and spleen are recognized as the preliminary indicators to reflect the body' immune function [15]. Previous studies have suggested that the crude extract of Cordyceps could inhibit the tumor growth associated with the elevated thymus and/or spleen index [26]. In this study, the thymus index of C57BL/ 6 mice and the spleen index of $\mathrm{KM}$ mice were increased when treated with CFCT of $100 \mathrm{mg} / \mathrm{kg}$ and $50 \mathrm{mg} / \mathrm{kg}$, respectively (Table 1 and Table 2). The results imply that CFCT might have different immune regulation mechanisms on two mouse models. The increased thymus index suggested that CFCT could potentiate cell-mediated immunity of C57BL/ 6 mice, and the increased spleen index implied that CFCT could enhance humor-mediated immunity of KM mice. However, a bit confusing fact is that the spleen index of $\mathrm{C} 57 \mathrm{BL} / 6$ mice and the thymus index of KM mice were slightly decreased under treatment with CFCT. Because the immune organ index is just a superficial indicator of immune function, the precise effects of CFCT on immune system need further investigation.

The presence of metastasis is the major cause of cancer mortality in millions of cancer patients, and it is urgently required to develop new anticancer agents with antimetastatic activities. Melanoma is one of the most aggressive skin cancers with a high metastatic potential, and it is difficult to be curbed [27]. In most cases, the lung is the first organ that tumor cells detaching from primary tumors encounter, making it a major site for tumor metastasis. In this study, the lung metastasis of melanoma B16F10 cells was effectively inhibited by CFCT alone and in combination with CTX, but its mechanism of action remained unclear. One group reported that the combination of chemotherapeutic agent methotrexate and water extract of C. sinensis (WECS) 


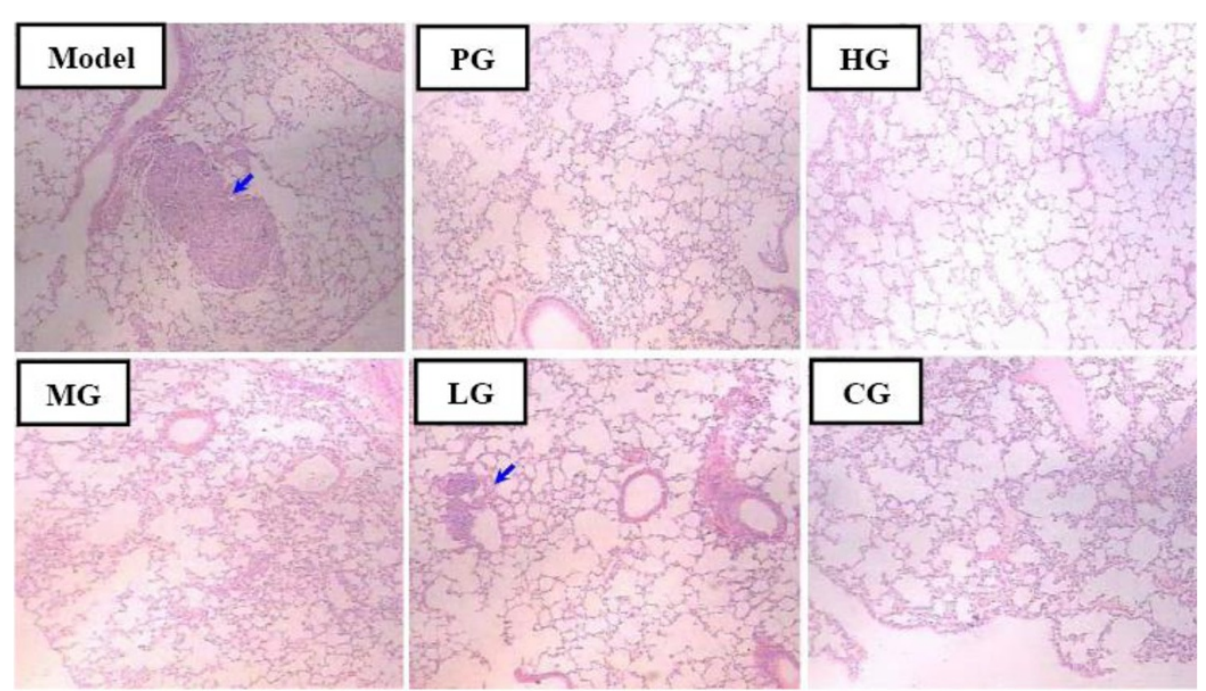

Fig. 5 Pathological changes of lung tissue from B16F10-bearing C57BL/6 mice by HE staining after administration. Blue arrows denote metastasis. All groups as described in Fig. 2

could inhibit the hematogenic lung metastasis in melanoma B16-BL6-bearing C57BL/6 J mice [28]. WECS could also reduce the hepatic metastasis of melanoma B16-F0 cells in C57BL/6Cr mice, and its mechanism of antimetastatic action was associated with reducing the hepatocyte growth factor, and accelerating the secretion of tissue inhibitor of metalloproteinase-1 [29, 30]. Another group demonstrated that exopolysaccharide of $C$. sinensis inhibited tumor growth and metastasis in the lungs and livers of B16 melanoma-bearing mice by reducing c-Myc, c-Fos, and vascular endothelial growth factor receptor (VEGF) expression levels [31]. Here VEGF

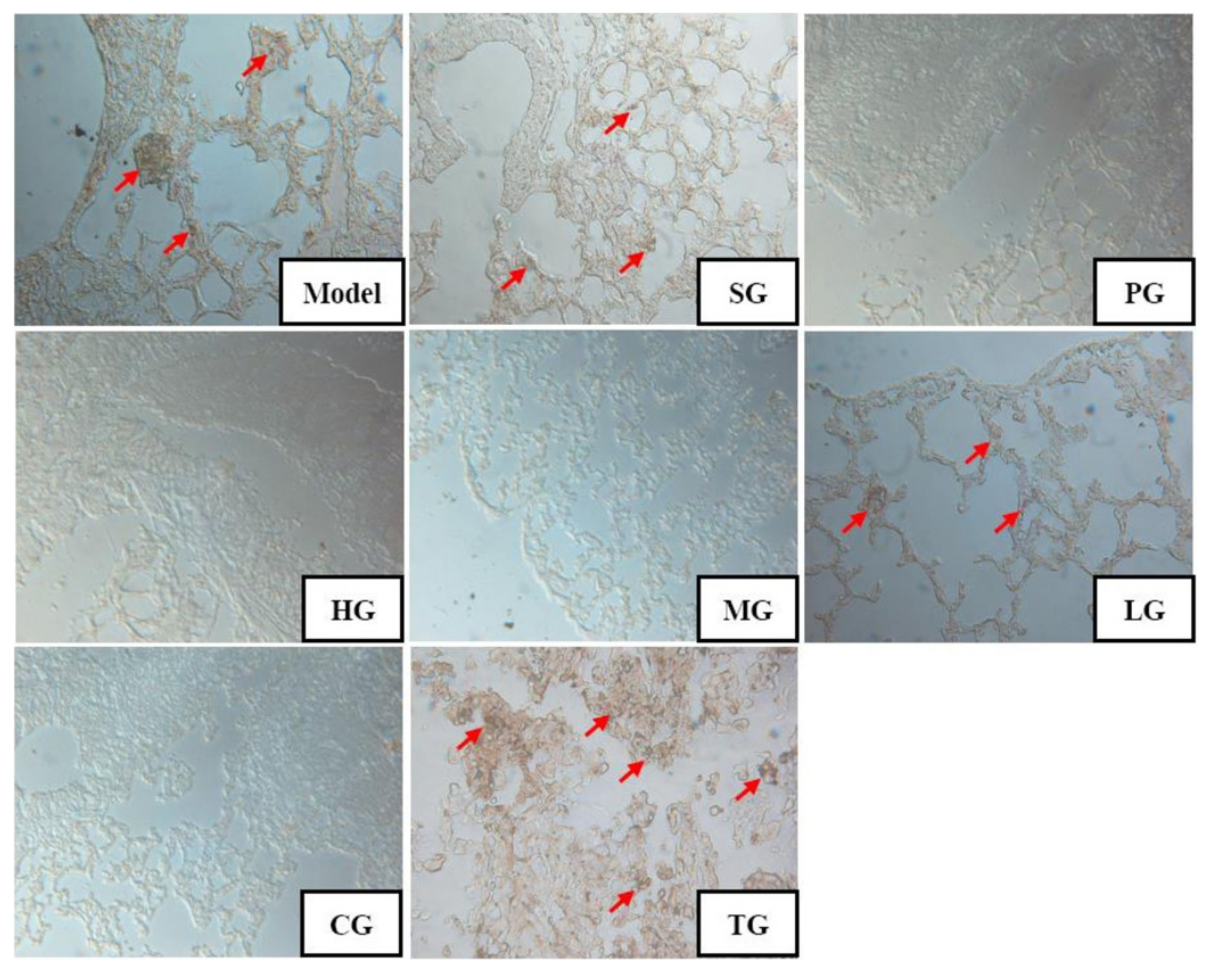

Fig. 6 Effects of CFCT on immunohistochemistry in lung tissue from melanoma B16F10-bearing C57BL/6 mice. Light micrograph of HMB45positive cells (original magnification $\times 400$ ) stained by the anti-mouse HMB45 mouse monoclonal antibody. Red arrows denote HMB45 protein. TG: solid tumor tissue of model group. Other groups as described in Fig. 2 


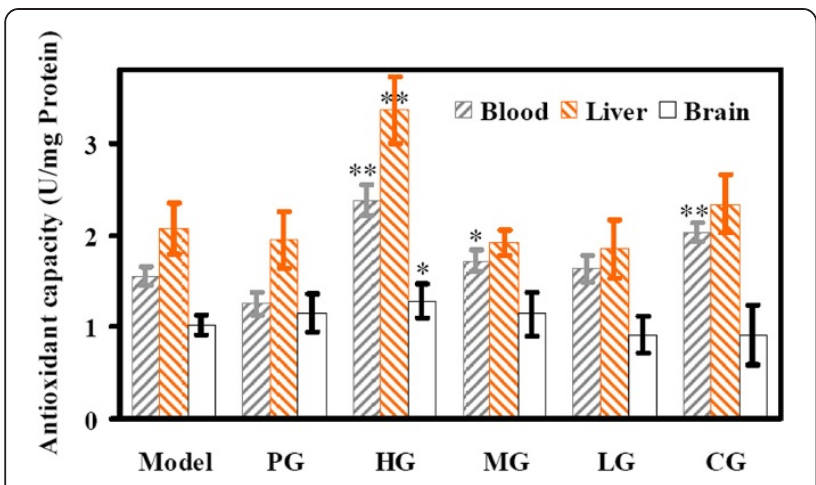

Fig. 7 Effects of CFCT on antioxidant ability in different tissues of melanoma B16F10-bearing C57BL/6 mice. ${ }^{*} P<0.05$, ${ }^{* *} P<0.01$ vs. model group. All groups as described in Fig. 2

is a central molecule involved in the angiogenesis and metastasis and regarded as a cancer therapeutic target of the angiogenesis and metastasis [32, 33]. Interestingly, Ruma et al. recently observed that $C$. militaris extract could remarkably suppress melanoma growth by downregulating VEGF expression and inducing the cell apoptosis [21]. This work implies that the anticancer and antimetastatic mechanisms of CFCT are possibly associated with the anti-angiogenicity and apoptosis induction.

It is well known that oxidative stress is involved in a variety of pathological processes, such as cancer, neurodegenerative diseases, ageing, and cardiovascular diseases $[34,35]$. The role of redox signaling in cancer progression is well accepted. In general, a higher level of reactive oxygen species (ROS) is essential for cancer cell survival, proliferation, and metastasis via the regulation of redox signaling. $\mathrm{H}_{2} \mathrm{O}_{2}$ can function as a signaling molecule in tumor angiogenesis by inhibiting tyrosine phosphatase activity in growth factor signaling transduction [36, 37]. Therefore, therapeutic strategies aimed at removal of free radicals, especially $\mathrm{H}_{2} \mathrm{O}_{2}$, might be a reasonable choice for the treatment and prevention of cancer. Recently many extracts and products of Cordyceps have been shown to have significant antioxidant properties [38]. Polysaccharides from C. sinensis and C. militaris possessed strong antioxidant activities such as scavenging free-radicals, inhibiting lipid peroxidation, chelating metal ion, etc. $[39,40]$. Our previous studies demonstrated that polysaccharides of $C$. jiangxiensis and $C$. taii possessed antioxidant activities in vitro and in vivo $[9,41]$. The facts suggested that Cordyceps may be a promising source of antioxidants or antioxidant compounds. In the case of B16F10-bearing mice treated with CFCT of $100 \mathrm{mg} / \mathrm{kg}$, the activities of GSH-Px, one of primary antioxidant enzyme that converts glutathione to oxidized glutathione and metabolizes $\mathrm{H}_{2} \mathrm{O}_{2}$ and lipid hydroperoxides into harmless products, were found to be significantly increased in blood, brain and liver tissues compared to the model mice (Fig. 7). Our data indicate that the antitumor activity of CFCT could be due to, at least in part, its ability to enhance the GSH-Px activity of organisms. Therefore, the antioxidant properties of CFCT in vitro and in vivo and whether the tumor inhibition of CFCT is linked to antioxidation are to be addressed here.

Previous studies demonstrated that the antitumor effective components of water extracts in C. sinensis and C. militaris included cordycepin and polysaccharide [21, 31, 42]. Chemical compositions of lipophlic extracts in Cordyceps species are more complicated and diversified. The definite antitumor effect of CFCT in vivo provided a substantial foundation for the further development of new chemopreventive lead compounds from C. taii. Recently, a bioassay-guided separation of CFCT was conducted by combining silica gel column chromatography and semipreparative high-performance liquid chromatography with SRB assay by our group [43]. Although cordycepin, the most well-known anticancer substance in Cordyceps, was not detected in C. taii [44], three known compounds - helvolic acid, cytochalasin $\mathrm{C}$ and zygosporin $\mathrm{D}$, and three new cytochalasin derivatives, were isolated and identified from CFCT $[13,43]$. Furthermore, we found that cytochalasin C, zygosporin $\mathrm{D}$ and three new cytochalasin derivatives displayed remarkable cytotoxicity against both lung cancer cell lines 95-D and A-549 in vitro, while no cytotoxic effect to human normal lung cells MCR-5. These findings indicated that those five compounds had excellent selectivity between normal and cancer cells, and their molecular mechanism of anticancer deserved an in-depth study [43]. Helvolic acid, a fusidane skeleton triterpenic acid with antimicrobial activity, was identified from Cordyceps species for the first time, and was also verified that it had potent cytotoxic activity against various human cancer cell lines [13]. Through these researches, the concrete effective components of CFCT could be understood at least partially, which is important to our searching for cancer chemopreventive compounds from the medicinal mushroom C. taii.

\section{Conclusion}

CFCT was found to have notable antitumor and antimetastatic activities in vivo. The possible mechanism of the anticancer effect of CFCT may be related to its antiproliferation, antioxidant, and immunoregulatory effects, but a further investigation is required. To our knowledge, this is the first report on antitumor and antimetastatic activities by the lipophlic extract of C. taii.

\section{Competing interests}

The authors declare that they have no competing interests. 


\section{Authors' contributions}

R-ML interpreted the experimental data and drafted the manuscript. X-JZ collected the experimental data. Y-FY performed the pathological analysis. G-YL participated the study design. J-JZ provided necessary advices and recommendations throughout the research. J-HX made substantial contributions to conception and design, and helped to draft the manuscript. All authors read and approved the final manuscript.

\section{Authors' information}

Joint first author is Xiao-Jie Zhang.

\section{Acknowledgments}

The authors are grateful to financial supports from the National Natural Science Foundation of China (No. 81060260; 81260278), Guizhou High-Level Innovative Talent Support Program (No. QKH-RC-20154028), Program for Innovative Research Team in Guizhou Province (No. QKH-RCTD-20134035), and the Joint Research Foundation of S\&T in Guizhou Province, (No. QKH-J-LKZ-2011-36).

\section{Author details}

'Guizhou Center for Translational Medicine \& Laboratory of Cell Engineering, Affiliated Hospital of Zunyi Medical University, Zunyi 563000, China. ${ }^{2}$ Department of Pathology, Affiliated Hospital of Zunyi Medical University, Zunyi 563000, China. ${ }^{3}$ State Key Laboratory of Microbial Metabolism, Joint International Research Laboratory of Metabolic \& Developmental Sciences, and School of Life Sciences \& Biotechnology, Shanghai Jiao Tong University, Shanghai 200240, China.

\section{Received: 12 April 2015 Accepted: 2 July 2015}

Published online: 09 July 2015

\section{References}

1. Rebecca S, Deepa N, Ahmedin J. Cancer statistics, 2013. CA-Cancer J Clin 2013;63(1):11-30.

2. Bernard WS, Christopher PW. World cancer report 2014. Lyon: IARC Press; 2014.

3. Green JA, Lainakis G. Cytotoxic chemotherapy for advanced or recurrent cervical cancer. Ann Oncol. 2006;17:230-2.

4. Niero EL, Rocha-Sales B, Lauand C, Cortez BA, de Souza MM, Rezende-Teixeira P, et al. The multiple facets of drug resistance: One history, different approaches. J Exp Clin Canc Res. 2014;33(1):37.

5. Zhong JJ, Xiao JH. Secondary metabolites from higher fungi: discovery, bioactivity, and bioproduction. Adv Biochem Eng Biotech. 2009;113:79-150

6. Xiao JH, Zhong JJ. Secondary metabolites from Cordyceps species and thei antitumor activity studies. Recent Pat Biotechnol. 2007;1(2):123-37.

7. Holliday J, Cleaver M. Medicinal value of the caterpillar fungi species of the genus Cordyceps (Fr.) Link (Ascomycetes). A review. Int J Med Mushrooms. 2008;10(3):219-34

8. Liang ZQ. Flora Fungorum Sinicorum, Cordyceps, Science Press, Beijing, China. 32nd ed. 2007.

9. Xiao JH, Xiao DM, Chen DX, Xiao Y, Liang ZQ, Zhong JJ. Polysaccharides from the medicinal mushroom Cordyceps taii show antioxidant and immunoenhancing activities in a D-galactose-induced aging mouse model. Evid-Based Compl Alt. 2012; doi:10.1155/2012/273435

10. Xiao JH, Xiao DM, Sun ZH, Xiong Q, Liang ZQ, Zhong JJ. Chemical compositions and antimicrobial property of three edible and medicinal Cordyceps species. J Food Agric Environ. 2009:7(3\&4):91-100.

11. Xiao JH, Xiao DM, Xiong Q, Liang ZQ, Zhong JJ. Optimum extraction and high-throughput detection of cordycepic acid from medicinal macrofungi Cordyceps jiangxiensis, Cordyceps taii and Cordyceps gunnii. J Food Agric Environ. 2009;7(3\&4):328-33.

12. Xiao JH, Xiao DM, Xiong Q, Liang ZQ, Zhong JJ. Nutritional requirements for the hyperproduction of bioactive exopolysaccharides by submerged fermentation of the edible medicinal fungus Cordyceps taii. Biochem Eng J. 2010;49:241-9.

13. Dou Y, Xiao JH, Xia XX, Zhong JJ. Effect of oxygen supply on biomass and helvolic acid production in submerged fermentation of Cordyceps taii. Biochem Eng J. 2013:81:73-9.

14. Lü YH, Pan WD, Xiao JH, Sun ZH, Zhong JJ. Cytotoxic mechanism of novel compound jiangxienone from Cordyceps jiangxiensis against cancer cells involving DNA damage response pathway. Process Biochem. 2014;49:697-705.
15. Alexander M, Steffen J. Development and function of dendritic cell subsets Immunity. 2014:40(5):642-56.

16. Kunimoto $R$, Jimbow $K$, Tanimura A, Sato M, Horimoto K, Hayashi T, et al. SIRT1 regulates lamellipodium extension and migration of melanoma cells. J Invest Dermatol. 2014;134(6):1693-700.

17. Teixeira TF, Gentile LB, Da Silva TC, Mennecier G, Chaible LM, Cogliati B, et al. Cell proliferation and expression of connexins differ in melanotic and amelanotic canine oral melanomas. Vet Res Commun. 2014;38(1):29-38.

18. Traverso N, Ricciarelli R, Nitti M, Marengo B, Furfaro AL, Pronzato MA, et al. Role of glutathione in cancer progression and chemoresistance. Oxid Med Cell Longev. 2013; doi: 10.1155/2013/972913

19. Suggitt M, Bibby MC. 50 years of preclinical anticancer drug screening: empirical to target-driven approaches. Clin Cancer Res. 2005;11(3):971-81.

20. Park SE, Kim J, Lee YW, Yoo HS, Cho CK. Antitumor activity of water extracts from Cordyceps militaris in $\mathrm{NCl}-\mathrm{H} 460$ cell xenografted nude mice. J Acupunct Meridian Stud. 2009;2(4):294-300.

21. Ruma IM, Putranto EW, Kondo E, Watanabe R, Saito K, Inoue Y, et al. Extract of Cordyceps militaris inhibits angiogenesis and suppresses tumor growth of human malignant melanoma cells. Int J Oncol. 2014;45(1):209-18.

22. Jeong MH, Lee CM, Lee SW, Seo SY, Seo MJ, Kang BW, et al. Cordycepinenriched Cordyceps militaris induces immunomodulation and tumor growth delay in mouse-derived breast cancer. Oncol Rep. 2013;30(4):1996-2002.

23. Mantovani A, Allavena P, Sica A. Cancer-related inflammation. Nature. 2008;454(7203):436-44.

24. Lee DK, Hakim FT, Gress RE. The thymus and the immune system: layered levels of control. J Thorac Oncol. 2010;5 Suppl 4:273-6.

25. Vincenzo B, Mikael JP. The spleen in local and systemic regulation of immunity. Immunity. 2013;39(5):806-18.

26. Paterson RRM. Cordyceps - A traditional Chinese medicine and another fungal therapeutic biofactory? Phytochemistry. 2008;69(7):1469-95.

27. Jemal A, Siegel R, Ward E, Hao Y, Xu J, Thun M. Cancer statistics, 2009. CA-Cancer J Clin. 2009:59(4):225-49.

28. Nakamura K, Konoha K, Yamaguchi Y, Kagota S, Shinozuka K, Kunitomo M. Combined effects of Cordyceps sinensis and methotrexate on hematogenic lung metastasis in mice. Receptor Channel. 2003;9(5):329-34.

29. Kubo E, Yoshikawa N, Kunitomo M, Kagota S, Shinozuka K, Nakamura K. Inhibitory effect of Cordyceps sinensis on experimental hepatic metastasis of melanoma by suppressing tumor cell invasion. Anticancer Res. 2010;30(9):3429-34.

30. Kubo E, Sato A, Yoshikawa N, Kagota S, Shinozuka K, Nakamura K. Effect of Cordyceps sinensis on TIMP-1 secretion from mouse melanoma cell. Cent Eur J Biol. 2012;7(1):167-71.

31. Yang JY, Zhang WY, Shi PH, Chen JP, Han XD, Wang Y. Effects of exopolysaccharide fraction (EPSF) from a cultivated Cordyceps sinensis fungus on c-Myc, c-Fos, and VEGF expression in B16 melanoma-bearing mice. Pathol Res Pract. 2005:201(11):745-50.

32. Saharinen P, Eklund L, Pulkki K, Bono P, Alitalo K. VEGF and angiopoietin signaling in tumor angiogenesis and metastasis. Trends Mol Med. 2011;17(7):347-62.

33. Dewing D, Emmett M, Pritchard JR. The roles of angiogenesis in malignant melanoma: trends in basic science research over the last 100 years. ISRN Oncol. 2012:2012:1-7.

34. Aboul-Enein HY, Berczynski P, Kruk I. Phenolic compounds: the role of redox regulation in neurodegenerative disease and cancer. Mini-Rev Med Chem. 2013;13(3):385-98

35. Yang WY, Zou LZ, Huang $\mathrm{CH}$, Lei YL. Redox regulation of cancer metastasis: molecular signaling and therapeutic opportunities. Drug Develop Res. 2014;75(5):331-41.

36. Gorrini C, Harris IS, Mak TW. Modulation of oxidative stress as an anticancer strategy. Nat Rev Drug Discov. 2013;12(12):931-47.

37. Wu WS. The signaling mechanism of ROS in tumor progression. Cancer Metast Rev. 2006;25(4):695-705

38. Ng TB, Wang HX. Pharmacological actions of Cordyceps, a prized folk medicine. J Pharm Pharmacol. 2005;57(12):1509-19.

39. Yu HM, Wang BS, Huang SC, Duh PD. Comparison of protective effects between cultured Cordyceps militaris and natural Cordyceps sinensis against oxidative damage. J Agr Food Chem. 2006:54(8):3132-8.

40. Dong $\mathrm{CH}, \mathrm{Yao}$ YJ. In vitro evaluation of antioxidant activities of aqueous extracts from natural and cultured mycelia of Cordyceps sinensis. Food Sci Technol. 2008:41(4):669-77.

41. Xiao JH, Xiao DM, Sun ZH, Xiao Y, Zhong JJ. Antioxidative potential of polysaccharide fractions produced from traditional Chinese medicinal 
macrofungus Cordyceps jiangxiensis in vitro. Afr J Biotechnol. 2011;10(34):6607-15.

42. Yoshikawa N, Nishiuchi A, Kubo E, Yamaguchi Y, Kunitomo M, Kagota S, et al. Cordyceps sinensis acts as an adenosine A3 receptor agonist on mouse melanoma and lung carcinoma cells, and human fibrosarcoma and colon carcinoma cells. Pharmacol Pharm. 2011;2(4):266-70.

43. Li XG, Pan WD, Luo HY, Liu RM, Xiao JH, Zhong JJ. New cytochalasins from medicinal marcofungus Crodyceps taii and their inhibitory activities against human cancer cells. Bioorg Med Chem Lett. 2015;25:1823-6.

44. Liu RM, Zhang XJ, Li XF, Xiao JH, Zhong JJ. Simple and rapid method for simultaneous determination of eleven nucleosides and nucleobases in medicinal macrofungus Cordcyeps taii. Lat Am J Pharm. 2015;34(2):283-90.

\section{Submit your next manuscript to BioMed Central and take full advantage of:}

- Convenient online submission

- Thorough peer review

- No space constraints or color figure charges

- Immediate publication on acceptance

- Inclusion in PubMed, CAS, Scopus and Google Scholar

- Research which is freely available for redistribution 